Vol. 6, n $1 \mid 2002$

Varia

\title{
Donald R. THOMAS, Detective Fiction and the Rise of
}

Forensic Science

Cambridge, Cambridge University Press, 1999, 341 p. ISBN 0-521-65303-7.

\section{Dominique Kalifa}

\section{(2) OpenEdition}

\section{Journals}

Édition électronique

URL : https://journals.openedition.org/chs/264

DOI : $10.4000 /$ chs. 264

ISSN : 1663-4837

Éditeur

Librairie Droz

Édition imprimée

Date de publication : 1 juillet 2002

Pagination : 136-13801/07/2002

ISBN : 2-600-00803-9

ISSN : 1422-0857

\section{Référence électronique}

Dominique Kalifa, «Donald R. Thomas, Detective Fiction and the Rise of Forensic Science », Crime, Histoire \& Sociétés / Crime, History \& Societies [En ligne], Vol. 6, n 1 | 2002, mis en ligne le 03 février 2009, consulté le 23 mars 2022. URL : http://journals.openedition.org/chs/264 ; DOI : https://doi.org/ $10.4000 /$ chs. 264

Ce document a été généré automatiquement le 23 mars 2022.

(c) Droz 


\title{
Donald R. THOMAS, Detective Fiction and the Rise of Forensic Science
}

Cambridge, Cambridge University Press, 1999, 341 p. ISBN 0-521-65303-7.

\author{
Dominique Kalifa
}

\section{RÉFÉRENCE}

Donald R. THOMAS, Detective Fiction and the Rise of ForensicScience, Cambridge, Cambridge University Press, 1999, 341 p. ISBN 0-521-65303-7.

Dans le sillage des travaux de Dennis Porter, de Glenn Most et surtout de Daniel Miller ${ }^{1}$, Donald Thomas poursuit dans cet ouvrage la réflexion réouverte depuis une vingtaine d'années sur la place et le sens du detective novel dans la société anglo-américaine. L'approche, qui entend penser ensemble les formes littéraires, l'évolution sociale et la signification politique, s'inscrit donc résolument dans le courant des cultural studies. Mais l'auteur privilégie un point d'entrée original, celui des liens tissés entre technologie et littérature, entre les diverses formes d'appareillage technique engendrées par la modernité policière et celles du roman de détection. À trois moments-clés du développement du genre (l'émergence dans les années 1840-1860, l'institutionnalisation dans les années 1890, l'âge d'or et les premières contestations de l'entre-deux-guerres), il propose donc d'examiner les relations qui s'établissent entre certaines innovations majeures comme le détecteur de mensonges, la photographie ou la dactyloscopie, et les textes canoniques de la tradition policière anglo-saxonne (de Poe à Chandler, en passant par Dickens, Wilkie Collins, Nathaniel Hawthorne, Conan Doyle, Mark Twain, Joseph Conrad, Agatha Christie et Dashiell Hammet).

2 De cette série d'analyses croisées se dégagent trois idées principales, qui constituent le principal apport du livre. La première concerne le caractère essentiellement textuel de l'opération policière. L'émergence conjointe des instruments de la police technique et du récit de détection repose en effet sur une même nécessité : transformer le corps de la victime ou du criminel en un texte susceptible d'être lu, déchiffré et interprété. Le 
stéthoscope, l'empreinte ou la photographie présentent en effet cette similitude avec le roman qu'ils textualisent le corps suspect. Et la dactyloscopie constitue l'achèvement du processus, puisque le texte révélateur est désormais écrit «à la main » sur le corps même du criminel. Pour partie empruntée aux concepts foucaldiens de "bio-pouvoir " et de "réseaux d'écritures", ce constat très sémiotique est, à défaut d'être neuf, subtilement mis en œuvre dans ce livre. Il nécessiterait cependant, pour être pleinement opératoire, d'être mis en relation avec les pratiques effectives de la lecture policière (c'est-à-dire avec les modalités concrètes de l'enquête judiciaire, qui ne se résument pas au $\mathrm{XIX}^{\mathrm{e}}$ siècle à quelques innovations scientifiques tardivement répandues), ainsi qu'avec une approche analogue du crime, considéré comme un acte illisible, soit parce qu'il existe en-deçà du langage, soit parce qu'il procède d'une stratégie d'effacement ou d'élision volontaire. La seconde idée concerne la capacité du roman, de par son essence herméneutique, à anticiper sur les innovations scientifiques pour mettre en scène des «technologies en devenir ». Du Cour révélateur d'Edgar Poe en 1843 aux usages pionniers de la photographie policière chez Dickens dès 1853, Thomas insiste à juste titre sur la nature expérimentale du détective novel et sur l'intense circulation à la fin du XIX siècle entre discours scientifique et fiction de grande consommation. Mais il montre également combien cet idéal se dissipe en période de doute ou de crise; ainsi la littérature hard-boiled des années 1930 (Hammet, Chandler, etc.) récuse-t-elle tout appel aux instruments de la vérité.

Mais c'est surtout la troisième idée développée par l'ouvrage qui se révèle la plus féconde. Thomas s'efforce en effet de montrer combien ces stratégies de textualisation du crime s'articulent aux anxiétés et aux exigences liées au processus de construction nationale. C'est généralement sous la forme d'un corps étranger, d'une sorte d'extériorité à la fois géographique, morale et politique, que le detective novel identifie le criminel : l'orang-outang de la rue Morgue, l'ouvrier français dans Bleak House de Dickens, l'Irlandais Moriarty chez Doyle, etc. Brute, bête ou monstre, le criminel est généralement un étranger, à tout le moins un être exotique, tandis que l'étranger, lui, est largement criminalisé. Soulignant les relations étroites qui lient les techniques d'identification policière avec les "savoirs" alors construits par l'anthropologie physique, le racialisme ou l'eugénisme (la figure de Galton est ici centrale), Thomas pointe l'importance de ces récits à la fois dans la constitution des identités personnelles, au cœur du processus de formation du sujet ou de l'individu moderne, et dans celle des identités nationales. C'est dans cette transformation de la personne en identité, c'est-à-dire en une série de signes matériels que l'on peut classer, archiver, objectiver, que résident selon lui l'enjeu et l'implication politiques des lectures policières.

4 La démonstration de Donald Thomas est à la fois habilement menée et convaincante. Certains points cependant invitent à la discussion. Constitué des œuvres les plus connues et les plus commentées de la littérature policière anglo-saxonne, le corpus étudié donne par endroit le sentiment d'un certain ressassement. Dans cet immense continent que constituent alors les romans criminels, d'autres choix, moins recouverts par la critique, pouvaient sans doute se révéler plus judicieux, tout en invitant à nuancer la thèse énoncée. Que les textes retenus appartiennent à deux traditions littéraires fort distinctes (anglaise et américaine), ce que l'auteur reconnaît volontiers, aurait également mérité d'être mieux pris en compte, notamment dans les développements consacrés à leur rôle dans l'édification des identités nationales. La naissance synchrone du genre dans d'autres États comme la France aurait aussi, dans 
cette perspective, mérité attention. On peut regretter également que la bibliographie utilisée demeure trop exclusivement anglo-saxonne. La lecture de la thèse pionnière de Régis Messac ${ }^{2}$, de l'essai du philosophe Allemand Siegfried Kracauer ${ }^{3}$, de la proposition de l'Italien Carlo Ginzburg sur le «paradigme indiciaire » (citée mais pas utilisée) ou de la très riche réflexion de l'Israélien Uri Eizensweig ${ }^{4}$ aurait sans doute donné à l'argumentation de Thomas une meilleure assise et une plus large portée.

\section{NOTES}

1. Dennis Porter, The Poursuit of Crime. Art and Ideology in Detective Fiction, New Haven/London, Yale University Press, 1983; Glenn W. Most, The Poetics of Murder. Detective Fiction and Literary Theory, New York, Harcourt, Brace Jovanovich,1983; Daniel A. Miller, The Novel and the Police, Berkeley, California University Press, 1988.

2. Régis Messac, Le « detective novel " et l'influence de la pensée scientifique, Paris, Champion, 1929.

3. Siegfried Kracauer, Le roman policier. Un essai philosophique (1929), rééd. Paris, Payot, 1972.

4. Uri Eizensweig, Le récit impossible. Sens et forme du roman policier, Paris, Bourgois, 1986.

\section{AUTEURS}

\section{DOMINIQUE KALIFA}

Université de Rennes 2-Haute Bretagne (France), kalifa@uhb.fr 